a blind and deaf man may know more, and may make greater contributions to knowledge, than one with sharp eyes and ears" refutes "the traditional view that our knowledge grows by the accumulation . . . of perceptions or observations" (my italics).

Again, Popper's eagerness to depreciate inductive learning leads him to claim (p.98) that in science "all new knowledge, . . . all discoveries, are the result of trial and error"' (rather than repetition). He even suggests (on p.50) that every author of an experimental paper should state in advance the specific hypothesis he intends to propose or discuss or test. This surely does less than justice to those phases of scientific investigation in which new territory has to be mapped before any non-trivial hypotheses can be formulated. Recording the first evoked brain potentials to a new stimulus, for example, may reveal nothing significant until one has made perhaps 100 repetitions; and what then emerges as an average out of the "background noise" may be a totally unexpected waveform which it would be merely impertinent and pretentious for the scientist to describe as a "test of his hypothesis".

The fact of the matter is that falsification of hypotheses is only one (albeit a highly desirable) way in which scientific information can be generated. True, we never start with a blank mind; but putting our eye to a new telescope, recording radio signals from distant stars or lifting a stone to see what lives underneath it are perfectly respectable ways of gaining scientific information even when we have only the vaguest of ideas as to what we may see. Until we have appropriate perceptual categories, we may indeed miss much that we may later learn to see in the same data; and unless we eventually find ourselves framing and testing hypotheses, our scientific enterprise will not have got very far. But in a new field especially, our scientific quest may have to begin by simply soaking ourselves for a time in raw data, letting our imagination play on them in the hope of gradually (or suddenly) spotting things to spur our curiosity and get the process of hypothesis-making and testing under way. Objective detachment in the scientific sense is entirely compatible with such imaginative commitment, since the object of the exercise is eventually to help all comers, whatever their values or attitudes, to reckon objectively $w^{\text {t th }}$ the reality we have encountered through it.

But let me not end on a negative note; for what distinguishes Popper from a great dull army of philosophers of science is that reading him is good for us. Even where he is (occasionally) outrageous he is at least sharp and clear enough for us to know better what we think in reaction; and at his best he is unsurpassed in the ability to recall to our minds the neglected emphases that make objective science worthy of our love. Yes, "love" is Popper's word for it:

I think that there is only one way to science - or to philosophy, for that matter: to meet a problem, to see its beauty and fall in love with it; to get married to it, and to live with it happily, till death do ye part - unless you should meet another and even more fascinating problem, or unless, indeed, you should obtain a solution. But even if you do obtain a solution, you may then discover, to your delight, the existence of a whole family of enchanting though perhaps difficult problem children for whose welfare you may work, with a purpose, to the end of your days [p.8]

Donald MacKay, a physicist, is Emeritus Professor of Communication and Neuroscience at Keele University.

\section{The view from Washington Square}

\section{Daniel D. Eley}

Electronic Processes in Organic Crystals.

By Martin Pope and Charles E.

Swenberg.

Oxford University Press: 1982. Pp.821. $£ 75, \$ 145$.

LYONS and Gutmann's Organic Semiconductors (Wiley, 1967) and Meier's book of the same title (Verlag Chemie, 1974) could reasonably be considered to present a comprehensive and balanced view of organic semiconductors. At that time, organic metals research was in its infancy, and organic superconductors still a gleam in the eye of W.A. Little. Any comparable treatment would these days need to account for at least 3,700 references, so Pope and Swenberg's disclaimer in their preface, that no attempt was made towards completeness nor objectivity in their choice of subject matter, must surely be understood.

How in fact is their book made up? The first four chapters, totalling 578 pages, deal with anthracene and its congeners, the last two with "materials of high dark conductivity" and "miscellaneous systems", a final ten-page postscript bringing us up to date on some nine topics such as solitons and superconductivity. At the outset one must record the general impression that the authors started with an enthusiastic exposition of their own main research topic of polycyclic aromatics, and only as an afterthought decided to include shorter reviews of some currently actively researched materials, inserting general theories where necessary; for example, the SchmidlinRoberts statistical treatment of donors and acceptors in dark conduction first appears on p.662 in connection with phthalocyanines.

In the four "anthracene chapters" Pope and Swenberg systematically discuss from a theoretical viewpoint optical properties, charge carrier generation as single positive or negative carriers or as pairs in the bulk, and photoemission, and give an authoritative account of the literature relevant to crystalline photoconductive molecular insulators. Complete beginners might find it helpful to first read Silinsh's introduction (Organic Molecular Crystals, published by Springer-Verlag in 1980) since there is a mass of detail and closely argued mechanisms to master. The book is largely free of typographical errors but one important one must be pointed out; in the energy level diagram on p.203, surely the valence and triplet state levels should be interchanged?

Turning to Chapter 5 , we read about radical-ion salts and charge-transfer complexes, transitions in one-dimensional systems, $(\mathrm{SN})_{x}$ and (TMTSF) ${ }_{2} \mathrm{PF}_{6}$ (the first organic superconductor at high pressure, updated to (TMTSF) ${ }_{2} \mathrm{ClO}_{4}$ with its ambient pressure superconductivity in the postscript). The following chapter includes phthalocyanines, polydiacetylenes, PVK and its TNF complex and various liquids. Perhaps rather strangely, the currently much-studied doped polyacetylene receives only three pages or so, with $\mathrm{AsF}$ s being first described as electron donor (p.700) and then as acceptor (p.701) bonus dormitat Homerus!

Oxford University Press advertise this book as suitable for second-year graduate students and specialists, a recommendation I would endorse with the proviso that the former in their first year should follow up the earlier historical development of the subject in one or other of the two monographs mentioned earlier. In his foreword Sir Nevill Mott describes the book as "massive and comprehensive", whereas massive and selective seems a more correct description; an equally long volume would be needed to deal with a whole range of topics omitted from this work. The problem here is that it is often the minority topics which contain the germs of future successes. Mott goes on to opine that "it is indeed open to doubt, in view of the widely divergent explanations published of some phenomena, whether a universally agreed upon model will ever emerge'".

Let us take heart from the fact that some 60 years separated the Van't Hoff-Le Bel tetrahedral carbon atom from the hybrid orbitals of Slater and Pauling, and a lot of valuable chemistry was carried out in the interval. It must be said that the need to set out so many physical theories has tended to obscure the simple physico-chemical guidelines which still help to direct organic synthesis to compounds with new electrical properties. Even if a unified physical theory must wait till the turn of the century, we may still hope for practical developments, useful materials to stand alongside PVK-TNF and justify our efforts. The present volume represents a valiant effort to bring order into an ever-expanding field, and help us all on our way.

Daniel D. Eley is Leverhulme Emeritus Fellow in the Department of Chemistry, University of Nottingham. 